\title{
Veterinary mycosis in a tropical country
}

\author{
Calderón-Hernández, A \& Urbina-Villalobos, A. \\ Mycology Laboratory, School of Veterinary Medicine, National University, Costa Rica
}

Objective: To share the experience of ten years of work in diagnosis in veterinary mycology and research on mycotic diseases in companion, productive and wildlife animals in Costa Rica.

Methods: This was a retrospective study carried out in our Laboratory between January 2008 and December 2017. Our database of fungal disease cases and research on veterinary mycology were included. The most relevant results of theses carried out by veterinary students are also presented. A mycotic disease was confirmed by direct microscopic identification of the organism in the clinical specimen and/or by histopathology and/or culture of the organism.

Results: For the study period there were a total of 2469 cases that underwent mycological analysis and $27.8 \%$ were confirmed with a mycotic disease. The most common mycosis was Malassezia spp. otitis and/or dermatitis with $36.1 \%$ (248/686), followed by dermatophytosis with $31 \%$ (213/686). Aspergillosis in poultry, in the third place, accounted for $26.7 \%$ (183/686) of the cases. Other systemic and subcutaneous mycosis were confirmed (Fig 1.). Research findings are summarized in Fig 2.

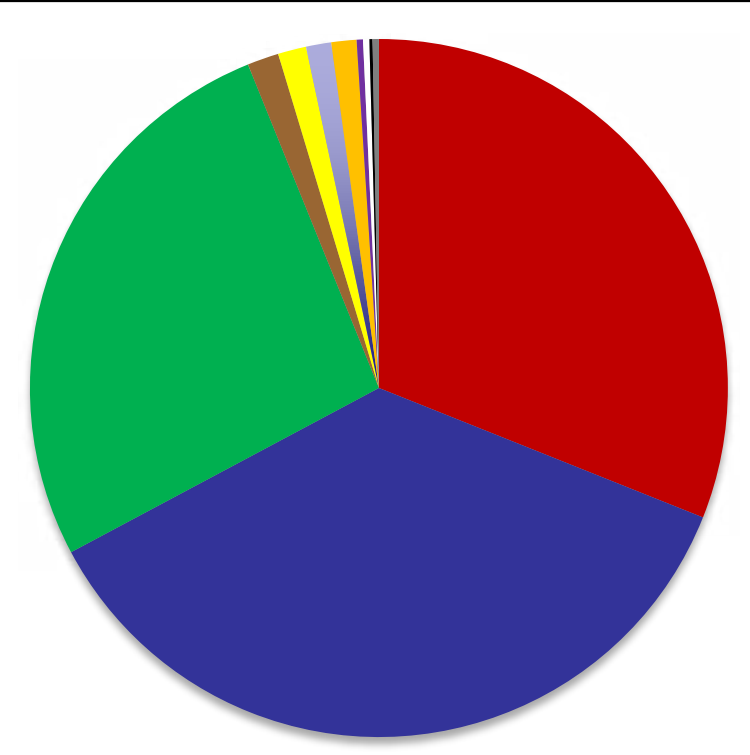
- Dermatophytosis
- Aspergillosis
Cryptococosis
Pythiosis
Hyphomycosis
- Conidiobolomycosis

Fig 1. Distribution of the mycosis diagnosed during 2008-2017

Conclusion: Superficial mycoses in dogs and cats are the most common diseases occurring all over the year in this tropical country. Variations in frequency and species could be the result of different geographic areas and living conditions of the animals. Due to climate conditions, Aspergillus spp. is widely prevalent and its control is a challenge for poultry industry. Confirmation of systemic and deep mycoses in wild and domestics animals have increased since veterinarians are more aware of the possibility of the mycotic etiology and are considering it in their differential diagnosis. Mycological surveys in fauna contribute to baseline knowledge on fungal agents and further studies are needed in order to elucidate threats to wildlife conservation.

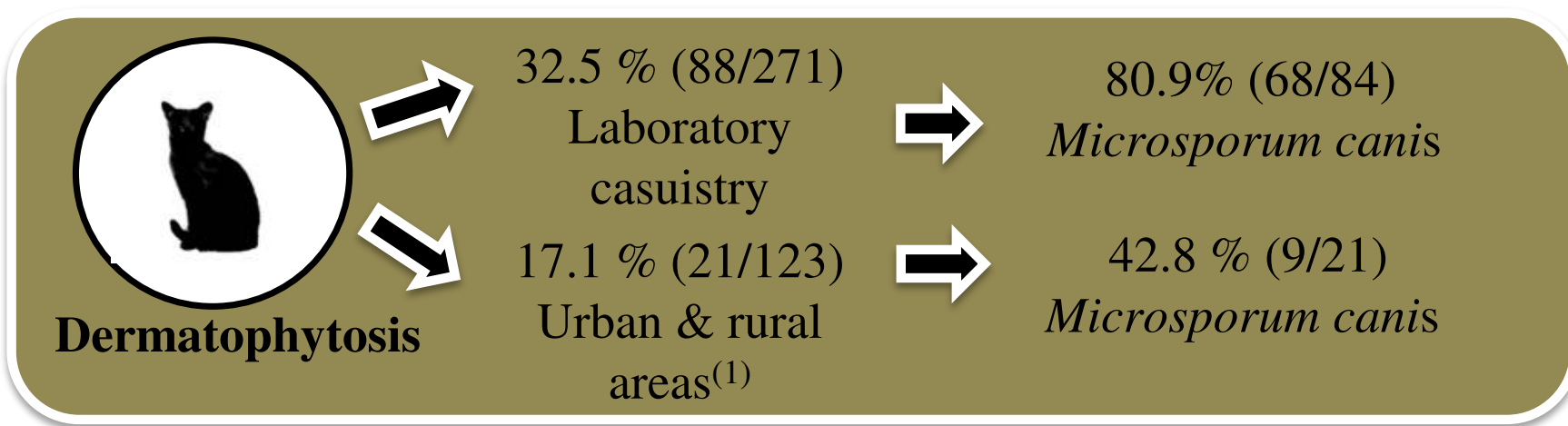

\begin{tabular}{|l|l|c|}
\cline { 2 - 3 } & \multicolumn{1}{|c|}{ Where? } & What? \\
\cline { 2 - 3 } & Laboratory casuistry & Microsporum canis \\
$11.3 \%(97 / 860)$ & $43.7 \%(28 / 64)$ \\
\cline { 2 - 3 } Dermatophytosis & Rural \& indigenous & Trichophyton \\
& areas $^{(2)} 20 \%(25 / 127)$ & mentagrophytes \\
& & $64 \%(16 / 25)$ \\
\cline { 2 - 3 } & Regional study ${ }^{(3)}$ & Microsporum gypseum \\
& $4.7 \%(6 / 127)$ & $83.3 \%(5 / 6)$ \\
\hline
\end{tabular}
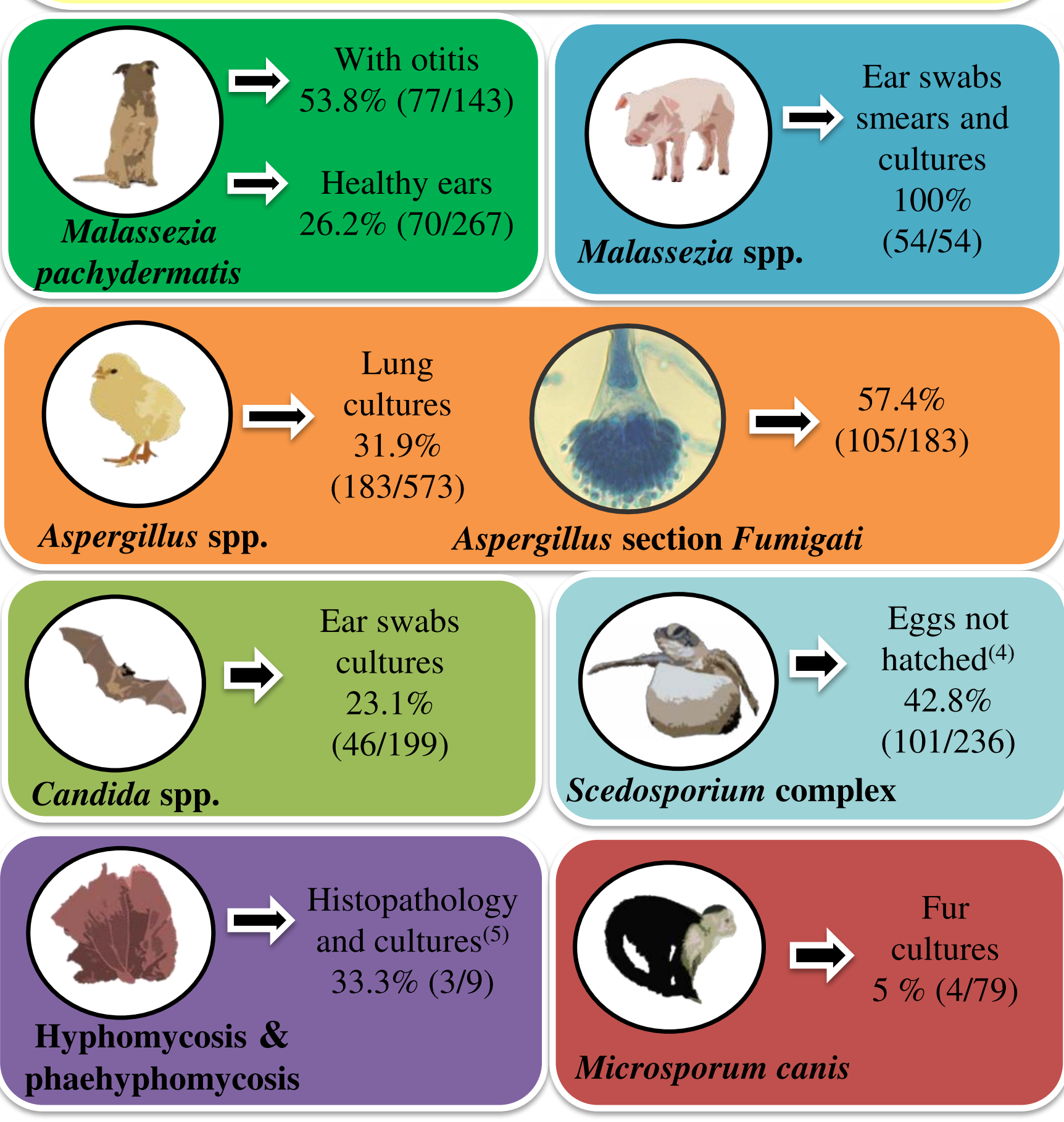

Fig 2. Main findings from mycologic surveys and theses carried out in our laboratory during 2008-2017

\section{References}

1. Solís-Jiménez, D. (2017). Veterinary Medicine Degree Thesis.

2. Acevedo-González, S. (2017). Veterinary Medicine Degree Thesis.

3. Arias-Carvajal, MG. (2013). Veterinary Medicine Degree Thesis.

4. Brenes-Chaves, LS. (2013). Master Degree Thesis.

5. Calderón-Hernández, A. (2017). Master Degree Thesis.

Acknowledgments to CONICIT and MICITT for funding this research and making this presentation possible, to the veterinary students who carried out their theses at the Laboratory and to the veterinary students assistants who collaborate in the maintenance of the laboratory databases. 